\title{
Power Saving MAC Protocols for WSNs and Optimization of S-MAC Protocol
}

Regular Paper

\author{
Simarpreet Kaur ${ }^{1 *}$ and Leena Mahajan² \\ 1 BBSBEC, Fatehgarh Sahib, Punjab \\ 2 IGCE, Abhipur, Punjab \\ *Corresponding author E-mail: simarpreet.kaur@bbsbec.ac.in
}

Received 19 April 2011; Accepted 02 June 2011

\begin{abstract}
Low power MAC protocols have received a lot of consideration in the last few years because of their influence on the lifetime of wireless sensor networks. Since, sensors typically operate on batteries, replacement of which is often difficult. A lot of work has been done to minimize the energy expenditure and prolong the sensor lifetime through energy efficient designs, across layers. Meanwhile, the sensor network should be able to maintain a certain throughput in order to fulfill the QoS requirements of the end user, and to ensure the constancy of the network. This paper introduces different types of MAC protocols used for WSNs and proposes S-MAC, a Medium-Access Control protocol designed for Wireless Sensor Networks. S-MAC uses a few innovative techniques to reduce energy consumption and support selfconfiguration. A new protocol is suggested to improve the energy efficiency, latency and throughput of existing MAC protocol for WSNs. A modification of the protocol is then proposed to eliminate the need for some nodes to stay awake longer than the other nodes which improves the energy efficiency, latency and throughput and hence increases the life span of a wireless sensor network.
\end{abstract}

Keywords Medium Access Control, Wireless Sensor Network, Energy Efficiency, Latency, Throughput.

\section{Introduction}

Wireless Sensor Networks have become one of the flourishing research fields in recent years, as they are intended to have wide applications in military, environmental, and many other fields [1]. Normally, the throughput, latency and the energy efficiency are unpredictable, and there exists a trade off among these measures. The objective of this work is to explore the maximum achievable throughput under certain network configurations and receiver structures, as well as optimum network designs that achieve the desired throughput, latency with minimal energy consumption.

Wireless Sensor Networks establish a special class of wireless data communication networks. A classic node in the WSN consists of a sensor, embedded processor, adequate amount of memory and transmitter/receiver circuitry. These sensor nodes are normally battery powered and they co-operate among themselves to perform a common task. Sensor Networks are the key to gathering the information needed by smart environments, whether in buildings, utilities, industrial, home, shipboard, transportation systems automation, or elsewhere. A Sensor Network is required that is fast and easy to connect and maintain. 
More and more wireless sensor networks are being used to gather information in real life applications [2].Looking toward to the future, the technology seems even more auspicious in two directions [3]. First, a few years from now more powerful wireless sensor devices will be accessible, and wireless sensor networks will have applicability in an endless number of scenarios, as they will be able to handle traffic loads not possible today, make more calculations, store more data, and live longer because of better energy sources. Second, a few years from now, the opposite scenario might also be possible. The availability of very forced, nano-technology made wireless sensor devices will bring a whole new world of applications, as they will be able to operate in environments and places unimaginable today. These two scenarios, at the same time, will both bring new research challenges that are always welcome to researchers.

The main goal of a WSN is to collect data from the environment and send it to a reporting site where the data can be observed and analyzed. Recent advances in sensor and wireless communication technologies in aggregation with developments in microelectronics have made available a new type of communication network made of battery-powered integrated wireless sensor devices. At present time, due to economic and technological reasons, most available Wireless Sensor devices are very self-conscious in terms of computational, memory, power, and communication capabilities [3]. This is the main reason why most of the research on WSNs has focused on the design of energy and computationally efficient algorithms and protocols, and the application domain has been restricted to simple data-oriented monitoring and reporting applications.

Medium Access Control (MAC) [4] is an important technique that permits the successful operation of the network. Medium Access Control for wireless sensor networks has been a very active research area in recent years. The old-style wireless medium access control protocol such as IEEE 802.11 is not suitable for the sensor network application because these are battery powered. The recharging of these sensors nodes is expensive and also not normally possible.

\section{Energy related Issues}

MAC sub-layer protocols for WSNs must address the following energy-related issues:

- Collisions: The collisions occur when two nodes transmit at the same time. The packets can get corrupted and it may be required to be retransmitted. So a lot of time and energy gets wasted during this transmission and reception. Collisions should be avoided because of the extra energy wasted in frame retransmission.
- Overhead: The other major problem is the Control Packet Overhead. These Control Packets do not contain any application data but are essential for the communication. The transmission and reception of these packets is overhead on the sensor network. Control messages and long headers in frames need to be avoided as much as possible, as they imply extra expensive communication costs.

- Overhearing: The other problem is overhearing in which a sensor node may receive packets that are not intended for it. This node could have turned off its radio to save its energy. Overhearing is the energy consumed by the nodes by being constantly listening and decoding frames that are not meant for them. This is a consequence of using a shared media in which nodes do not know a priori whether the transmissions are for them or not.

- Idle listening: Idle listening refers to the energy expended by the nodes by having their circuits $\mathrm{ON}$ and ready to receive while there is no activity in the network. This is particularly important in WSNs, as nodes use the channel sporadically. Strategies to turn nodes $\mathrm{ON}$ and OFF are very important in WSNs. The idle listening problem in wireless networks can be minimized by putting the radio into sleep mode.

- Complexity: Complexity refers to the energy expended as a result of having to run computationally expensive algorithms and protocols. One of the most important design goals in WSNs is therefore simplicity.

The other important characteristics of the Wireless Sensor Network are fairness, latency, throughput and bandwidth.

\section{WSN Medium Access Control Protocols}

Many medium access control (MAC) protocols for wireless sensor networks have been planned in the recent years. Most of these protocols have energy safeguarding as an objective. The pattern of energy use in the sensor nodes, however, depends on the nature of the application.

The MAC techniques proposed for WSNs can be divided into two categories namely Contention-based and Schedule based. Schedule based protocol can avoid collisions, overhearing and idle listening by scheduling transmit and listen periods but have strict time synchronization requirements.

The contention based protocol [5] on the other hand relax time synchronization requirements and easily adjust to the topology changes as some new nodes may join and other may die few years after deployment. These protocols are based on Carrier Sense Multiple Access (CSMA) technique and have higher costs for message collisions, overhearing, and idle listening. 
Normal

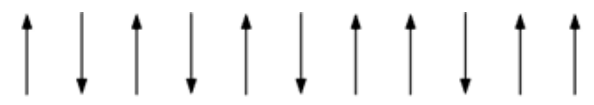

S-MAC

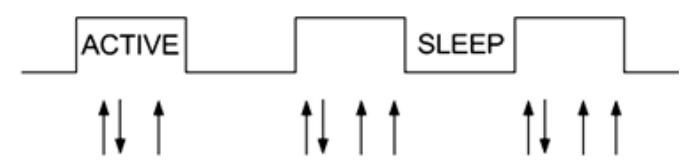

T-MAC

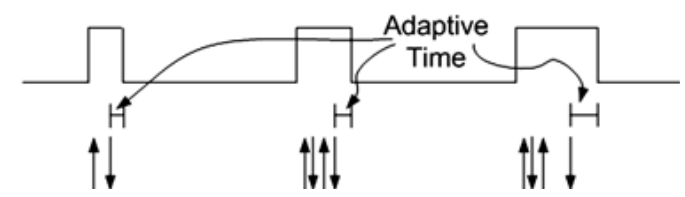

Figure 1. The S-MAC and T-MAC Protocols

IEEE 802.11 DCF (Distributed Coordination Function) is a contention based MAC protocol that is mainly built on the MACAW and widely employed in early WSN applications [6].

This section describes a number of energy-efficient MAC protocols for WSNs and states their contributions toward addressing their main issues such as overhearing, idle listening, control packet head and collision avoidance. Medium Access Control for wireless sensor networks has been a very energetic research area in recent years.

\subsection{Power Aware Multi-Access with Signaling (PAMAS) Protocol}

The Power Aware Multi-Access with Signaling (PAMAS) protocol [3] is based on the MACA protocol but includes a separate signaling channel to avoid the collisions and overhearing problems. All nodes utilize the signaling channel to exchange RTS-CTS frames and therefore gain access to the media. All nodes know who has gained the media and for how long, information that nodes use to turn themselves off. The other channel is used exclusively to transmit data frames, which is collision-free.

The main disadvantage of PAMAS is that it needs an additional radio for the signaling channel, which adds to the cost of sensor network devices.

\subsection{Sensor MAC (S-MAC) Protocol}

The Sensor MAC (S-MAC) protocol was introduced in [3] to solve the energy consumption related problems of idle listening, collisions, and overhearing in WSNs using only one transceiver. S-MAC considers that nodes do not need to be awake all the time given the low sensing event and transmission rates. A contention based S-MAC protocol is based on CSMA/CA, energy conservation and selfconfiguration are primary goals, while per-node fairness and latency are less important. To provide energy conservation, the S-MAC protocol tries to reduce undesirable energy depletion due to collision, overhearing, packet overhead and idle listening as well as it turns the radio on and off based on the fixed duty cycles.

The main drawback of S-MAC [5] is that the use of fixed duty cycles can waste considerable amounts of energy since the communication subsystem is activated even though no communication will take place.

\subsection{Time-out MAC (T-MAC) Protocol}

Timeout T-MAC [7] is the protocol based on the S-MAC protocol in which the active period is pre-empted and the sensor goes to the sleep period if no activation event has occurred for a time. The event can be reception of data, start of listen/sleep frame time etc. The Timeout-MAC (TMAC) protocol [3] introduces the idea of having an adaptive active/inactive (listening/sleeping) duty cycle to minimize the idle listening problem and improve the energy savings over the classic CSMA and S-MAC fixed duty cycle-based protocols. The T-MAC protocol, however, suffers from the known early sleep problem, which can reduce throughput.

\subsection{Wise MAC Protocol}

The Wise MAC [1] protocol which combines TDMA and CSMA techniques determines the length of the preamble dynamically to reduce the power consumption and thus it results better performance under especially variable traffic conditions as shown in figure 2.

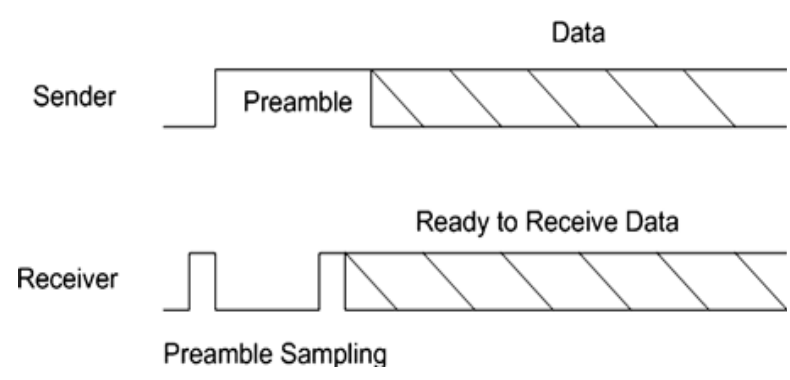

Figure 2. The preamble sampling technique used in wise MAC

Wise MAC performs better than one of the S-MAC variants [3]. Besides, its dynamic preamble length adjustment results in better performance under variable traffic conditions.

Main drawback of Wise MAC is that decentralized sleeplisten scheduling results in different sleep and wake-up times for each neighbour of a node [8]. This is especially an important problem for broadcast type of communication, since broadcasted packet will be buffered for neighbours in sleep mode and delivered many times as each neighbour wakes up. However, this redundant transmission will result in higher latency and power consumption. In addition, the hidden terminal problem comes along with Wise MAC model as in the 
Spatial TDMA and CSMA with Preamble Sampling algorithm. That is because Wise MAC is also based on nonpersistent CSMA. This problem will result in collisions when one node starts to transmit the preamble to a node that is already receiving another node's transmission where the preamble sender is not within the range.

\subsection{Berkeley Media Access Control (B-MAC) Protocol}

The Berkeley Media Access Control (B-MAC) protocol [3] is a CSMA based MAC protocol for WSNs. B-MAC introduces several interesting mechanisms and features. One mechanism is the Clear Channel Assessment (CCA) for effective collision avoidance, which takes samples of the media to estimate the noise floor. B-MAC also utilizes a preamble sampling-like technique called Low Power Listening (LPL) to minimize the idle listening problem. Finally, B-MAC includes the use of ACK frames for reliability purposes and throughput improvement. One of the most interesting features of B-MAC not available in any other protocol thus far is the capability of tuning its operation and mechanisms. B-MAC provides interfaces that can include/exclude B-MAC mechanisms, such as the CCA, acknowledgments, and LPL to trade-off power consumption, latency, throughput, fairness or reliability.

\subsection{Low Energy Adaptive Clustering Hierarchy (LEACH) Protocol}

The Low Energy Adaptive Clustering Hierarchy (LEACH) protocol [3] includes several ideas to reduce the energy consumption in WSNs. First, LEACH includes the idea of clustering but without including any powerful node as the cluster head. Second, in order to save additional energy in the nodes, LEACH utilizes a TDMA schedule-based MAC mechanism for intra-cluster communications.

A TDMA MAC protocol avoids collisions, the hidden and exposed terminal problems, and overhearing and idle listening problems by allowing nodes to turn themselves $\mathrm{ON}$ and OFF at appropriate times (given by the schedule) [3]. In LEACH there is no inter-cluster communications, instead cluster heads are meant to transmit directly to the sink node. In order to avoid collisions, LEACH utilizes CDMA for cluster head-sink communication, so cluster heads can transmit simultaneously to the sink without colliding with each other. It has some drawbacks. First, the cluster heads have to perform very computationally difficult and energy consuming tasks, such as preparing and managing the TDMA schedule, fusing and aggregating the data, and transmitting directly to the sink node. Second, LEACH needs synchronization, so that the TDMA scheme can actually work. Lastly, LEACH lacks multi-hop routing capabilities, which limit its applicability to small spaces.

\subsection{Traffic-Adaptive Medium Access (TRAMA) Protocol}

The Traffic-adaptive medium access (TRAMA) protocol [3] is a distributed TDMA mechanism that allows for flexible and self-motivated scheduling of time slots. TRAMA provides the energy-saving advantages of schedule-based mechanisms without the disadvantages of having a node as the main controller. TRAMA consists of three components that assign time slots only to stations that have traffic to send while being implemented in a distributed fashion. Further, it performs better than contention-based mechanisms because the slot assignment avoids collisions. TRAMA nodes use the Neighbor Protocol (NP) and the Schedule Exchange Protocol (SEP) to send their transmission schedules along with information related to the current time slot, one and twohops away node identifiers, and traffic interests. Then, the Adaptive Election Algorithm (AEA) uses this information to determine the transmitters and receivers for the current time slot and derive the node's sleep schedule.

\subsection{Self-Organizing Medium Access Control for Sensor Networks (SMACS) Protocol}

The Self-Organizing Medium Access Control for Sensor Networks (SMACS) Protocol was introduced in [3] as the protocol in charge of network startup and link layer organization in a series of protocols proposed to perform organization, routing, and mobility (ORM) functions in wireless sensor networks. SMACS is a distributed infrastructure-building protocol based on a neighborhood discovery procedure and the establishment and exchange of transmission schedules.

The DSMAC adds dynamic duty cycle feature to SMAC to achieve better latency for time sensitive applications. In DMAC [8] protocol, that can be considered as an improved version of Slotted Aloha, the primary goal is not only the energy conservation but also achieving lower latency.

\section{Related Work}

\subsection{Proposed S-MAC Protocol Design}

This paper suggests S-MAC, a medium-access control (MAC) protocol designed for wireless sensor networks. A new protocol is proposed to improve the energy efficiency, latency and throughput of existing MAC protocol for WSNs. One mechanism used to reduce energy expenditure is to periodically turn off the radio receivers of the sensor nodes in a coordinated manner. S-MAC may require some nodes to follow multiple sleep schedules causing them to wake up more often than other nodes [3]. A modification of the protocol is then proposed to eliminate the need for some nodes to stay awake longer than the other nodes. The modified version improves the energy efficiency, latency and the throughput and hence increases the life span of a wireless sensor network. 
Wireless sensor networks use battery-operated computing and sensing devices [7]. We expect sensor networks to be deployed in an ad hoc fashion, with nodes remaining largely inactive for long time, but becoming suddenly active when something is detected. These characteristics of sensor networks and applications motivate a MAC that is different from traditional wireless MACs such as IEEE 802.11 in several ways [6, 9]. Energy conservation and selfconfiguration are primary goals, while per-node fairness and latency are less important. S-MAC uses a few novel techniques to reduce energy consumption and support selfconfiguration. It enables low-duty-cycle operation in a multihop network. Nodes form virtual clusters based on common sleep schedules to reduce control overhead and enable traffic-adaptive wake-up. S-MAC uses in-channel signaling to avoid overhearing unnecessary traffic. Finally, S-MAC applies message passing to reduce contention latency for applications that require in-network data processing.

\subsection{S-MAC Protocol}

The Sensor MAC (S-MAC) protocol was introduced in [3] to solve the energy consumption related problems of idle listening, collisions, and overhearing in WSNs using only one transceiver. S-MAC considers that nodes do not need to be awake all the time given the low sensing event and transmission rates. S-MAC [7] reduces the idle listening problem by turning the radio OFF and ON periodically. Nodes are synchronized to go to sleep and wake up at the same time.

In order to address the issue of synchronization over multihop networks, nodes broadcast their schedules to all its neighbors. This is performed sending a small SYNC frame with the node schedule periodically. S-MAC divides time in two parts: the active (listening) part and the inactive (sleeping) part. The active part is divided at the same time in two time slots. During the first time slot, nodes are expected to send their SYNC frames to synchronize their schedules. The second time slot is for data transmission in which the SMAC protocol transmits all frames that were queued up during the inactive part. In order to send SYNC frames over the first time slot or RTS-CTS-DATA-ACK frames over the second time slot, nodes obtain access to the media utilizing the same contention mechanism included in IEEE 802.11, which avoids the hidden terminal problem and does a very good job avoiding collisions too. However, nodes using the IEEE 802.11 protocol waste a considerable amount of energy listening and decoding frames not intended for them [9]. In order to address this problem, S-MAC allows nodes to go to sleep after they hear RTS or CTS frames. During the sleeping time, a node turns off its radio to preserve energy.

Advantages: The energy wastage caused by idle listening is reduced by sleep schedules. In addition to its implementation simplicity, time synchronization overhead may be prevented with sleep schedule announcements [5].
Disadvantages: Broadcast data packets do not use RTS/CTS which increases collision probability. Adaptive listening incurs overhearing or idle listening if the packet is not destined to the listening node. Sleep and listen periods are predefined and constant, which decreases the efficiency of the algorithm under variable traffic load.

\subsection{Problems with S-MAC}

The following two problems have been identified in SMAC [7] protocol with multiple schedules.

1. Longer listen period

2. Sleep delay

\subsubsection{Longer Listen Period}

While choosing and maintaining the listen and sleep schedule, some nodes may have to keep wake during the listen time of more than one schedule [7]. This happens, for example, if a node, when it starts up, finds some of its neighbors following one schedule and the rest following another. The nodes following a shared schedule are said to form a virtual cluster. Figure 3 shows an example of this situation. Before node $M$ starts up, two isolated virtual clusters of nodes exist. Nodes $A, B$ and $C$ follow one schedule (schedule 1); and nodes $\mathrm{X}, \mathrm{Y}$ and $\mathrm{Z}$ follow another schedule (schedule 2). The circle around a node indicates the communication range of the node. When $\mathrm{M}$ starts, during its initial listening span - a synchronization period, it receives sync frames corresponding to both the schedules. $\mathrm{M}$ will then adopt one of the schedules (e.g. schedule 2) as its own, and announce this schedule in its sync frames. However, it will also have to wake up during the listen time of the other schedule. Thus $M$ has higher duty cycle, and consumes more energy.

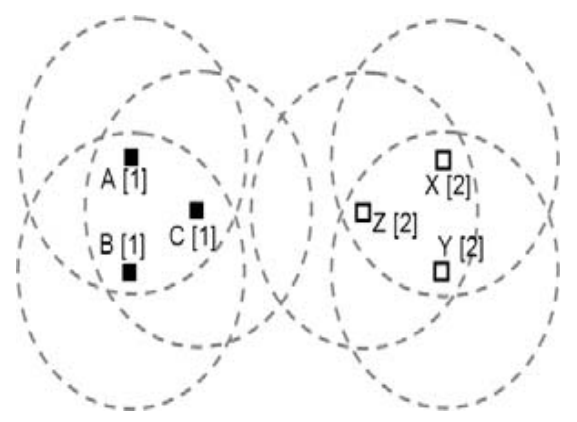

A : Before

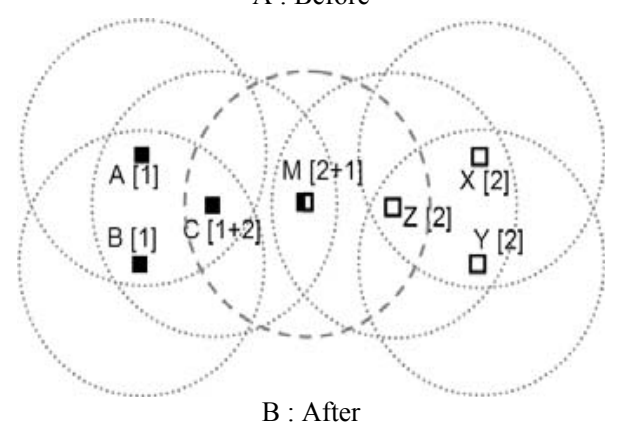

Figure 3. Sleep Schedule before and after node $\mathrm{M}$ join the network 


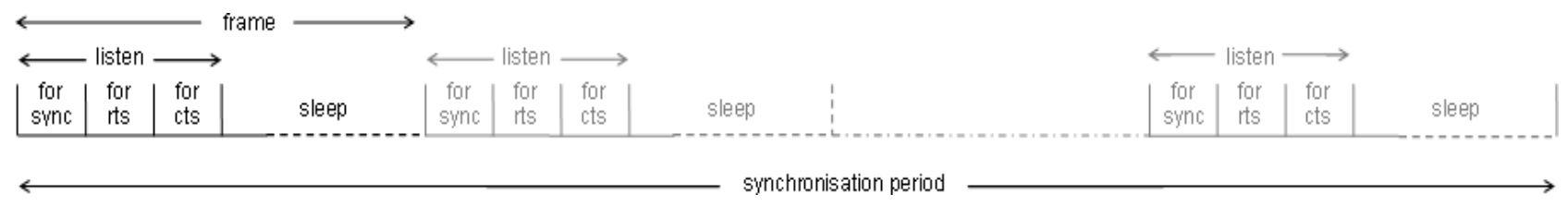

Figure 4. S-MAC Frame

\subsubsection{Sleep Delay}

Sleep delay introduces extra end to end delay called sleep delay [7]. Sleep delay increases communication latency in multi-hop networks, as intermediate nodes on a route do not necessarily share a common schedule. In a nutshell, the difficulty is to make a tradeoff between sleep delay and optimal active periods.

\subsection{Proposed Modification in S-MAC}

In this section, we propose an alteration of the S-MAC protocol. The modified protocol requires that when connectivity is established between two (or more) isolated virtual clusters (each following an independently chosen schedule) due to the introduction of a new node in the common neighborhood of the clusters, all nodes of these clusters form a single cluster by adopting the schedule of one of the clusters. This process of merger of clusters ensures that, except for the short period when clusters are merging, nodes follow exactly one cycle, avoiding the problems associated with multiple schedules. All nodes start with the same duty cycle. The nodes can increase their duty cycle by adding extra active periods when they require less latency or when they observe an increasing traffic load. Nodes can also decrease their duty cycle by removing the added active periods when there is less traffic. Active periods never get changed; nodes only insert their new active schedules in the middle of the sleep period.

\subsection{Modification in Frame Format}

The modified version of S-MAC presented here requires an additional field in the sync frame to hold the identifier of the schedule followed by the sender [7]. Every sync frame not only identifies the sender, it also identifies the schedule followed by the sender. This is the only modification required in the format of the S-MAC frames.

\section{Results and Discussion}

The modified protocol, S-MAC, removes the need for any node to follow multiple schedules. The nodes which would have depleted their energy because of having to follow multiple schedules under S- MAC are expected to last longer under S-MAC. The objective of this conversation is to compare the S-MAC and the modified proposed S-MAC protocol in terms of energy efficiency, latency and throughput. All the wireless sensor network MAC protocols have been compared in terms of energy efficiency, latency and throughput Simulation results of the WSN models are presented under varying network load conditions followed by performance comparisons and analysis.

The energy efficiency of the sensor nodes can be defined as the total energy consumed / total bits transmitted [4]. The average packet latency is the average time taken by the packets to reach the sink node. The sources of latency are carrier sense delay which is introduced when the sender performs carrier sense and its value is determined by the contention window size. Back off delay happens when carrier sense failed, either because the node detects another transmission or because collision occurs. Transmission delay is determined by channel bandwidth, packet length and the coding scheme adopted. Processing delay; the receiver needs to process the packet before forwarding it to the next hop. Propagation delay is determined by the distance between the sending and receiving nodes. Queuing delay depends on the traffic load. In the heavy traffic case, queuing delay becomes a dominant factor. The network throughput is defined as the total number of packets delivered at the sink node per time unit.

\subsection{Measurement of Energy Consumption}

Because of the difficulty in renewing or replacing the battery of each node in a wireless sensor network, the energy efficiency of the system is a major issue [8]. There are different ways to reduce energy consumption; one of them is to have a MAC protocol, specifically designed for this goal.

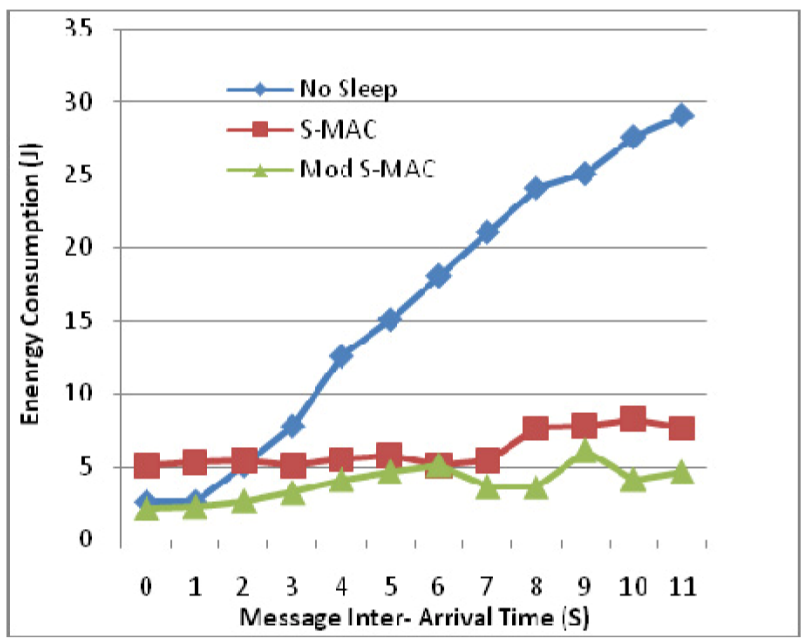

Figure 5. Energy Consumption 


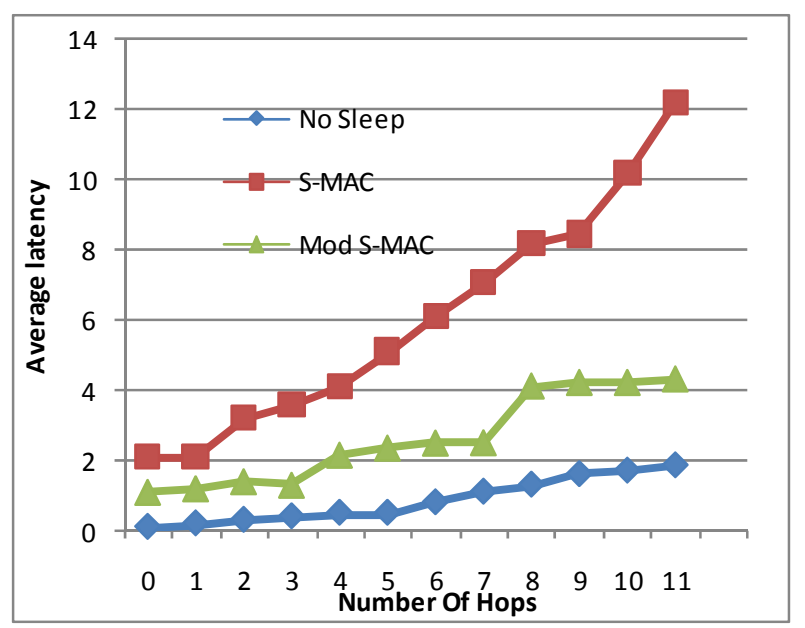

Figure 6(A). Average Message Latency under the lowest traffic load

The energy consumption was measured in the ten-hop network. In each test, the source node sends a fixed amount of data, 20 messages of 100-bytes each. Figure 5 shows that S-MAC with periodic sleep consumes much more energy over MAC without sleep, but the proposed MAC achieves better energy efficiency than the S-MAC protocol.

\subsection{Measurement of Average Message Latency}

Since S-MAC makes the trade-off of latency for energy savings, we expect that it can have longer latency under both the high and low traffic loads due to the periodic sleep on each node as shown in figure 6(A) and figure 6(B).

We considered two extreme traffic conditions, the lowest traffic load and highest traffic load. Under the lowest traffic load, the second message is generated on the source node after the first one is received by the sink. To do this, a coordinating node is placed near the sink. When it hears that the sink receives the message, it signals the source directly by sending at the highest power. In this traffic load, there is no queuing delay on each node. Compared with the MAC without sleep, the extra delay is only caused by the periodic sleep on each node. Under the highest traffic load, all messages are generated and queued on the source node at the same time. So, there is a maximum queuing delay on each node including the source node. The latency of the proposed MAC protocol is nearly equal to that of MAC without periodic sleep but still it doesn't reach the shortest latency.

\subsection{Measurement of Throughput}

Wireless Sensor Network should be able to maintain a certain throughput (which is equivalent to a certain delay constraint), in order to fulfil the QoS requirement of the end user, and to ensure the stability of the network [5]. Typically, the throughput and the energy efficiency are

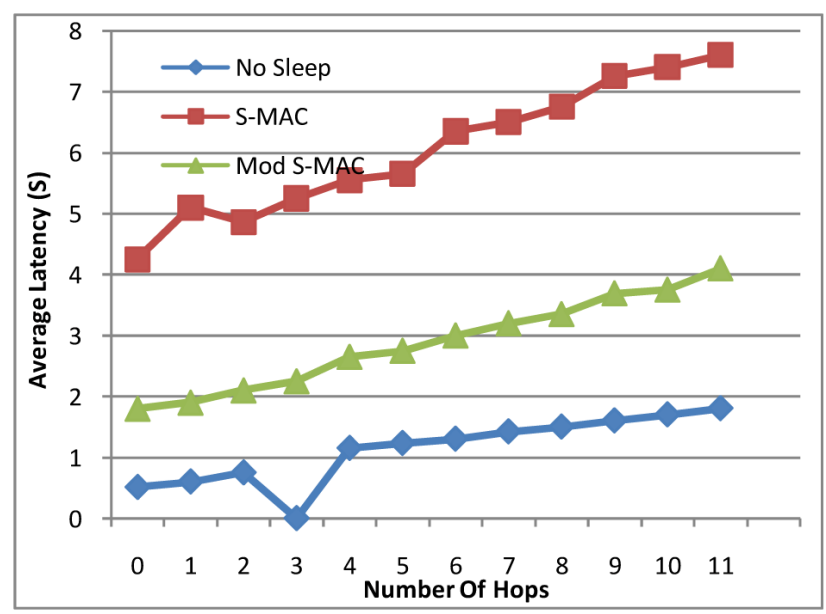

Figure 6(B). Average Message Latency under the highest traffic load

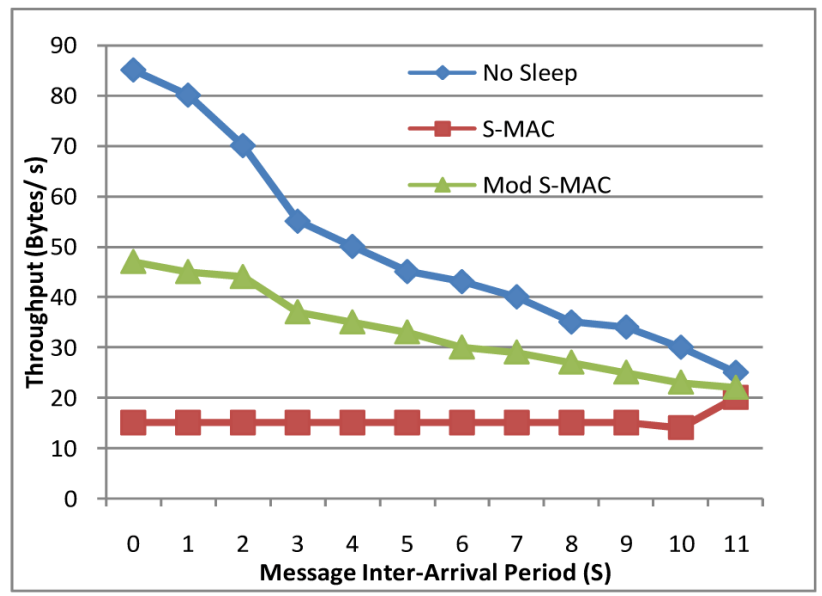

Figure 7. Throughput over 10-hops under varying traffic loads.

Just as S-MAC may increase latency, it may also reduce the throughput. Therefore we next evaluate throughput in the same 10-hop network. We first consider throughput for the highest traffic load, which is the same as that when measuring the latency in the highest traffic load. It delivers the maximum possible number of bytes of data in a unit time. The results in figure 7 show that for S-MAC as well as for proposed S-MAC, throughput drops as the number of hops increases, due to the RTS/CTS contention in the multi-hop network.

\subsection{Comparison of MAC Protocols}

Table I represents a comparison of MAC protocols investigated. Although there are various MAC layer protocols proposed for Wireless Sensor Networks, there is no protocol accepted as a standard. One of the reasons behind this is the MAC protocol choice will, in general, be application- dependent, which means that there will not be one standard MAC for sensor networks. All the MAC protocols for wireless sensor networks have been compared among themselves based on the energy efficiency, latency and throughput. It is observed from 


\begin{tabular}{|c|c|c|c|c|}
\hline Protocol & Type & $\begin{array}{c}\text { Energy } \\
\text { Efficiency }\end{array}$ & Latency & Throughput \\
\hline S-MAC & $\begin{array}{c}\text { Contention } \\
\text { based }\end{array}$ & Medium & Low & Low \\
\hline T-MAC & $\begin{array}{c}\text { Contention } \\
\text { based }\end{array}$ & $\begin{array}{c}\text { High under } \\
\text { variable } \\
\text { traffic }\end{array}$ & High & Low \\
\hline Wise-MAC & $\begin{array}{c}\text { Contention } \\
\text { based }\end{array}$ & Medium & High & Medium \\
\hline DMAC & $\begin{array}{c}\text { Contention } \\
\text { based }\end{array}$ & Low & Low & High \\
\hline TRAMA & $\begin{array}{c}\text { Contention } \\
\text { based }\end{array}$ & Low & Low & High \\
\hline Modified & $\begin{array}{c}\text { Contention } \\
\text { based }\end{array}$ & High & Medium & Medium \\
\hline
\end{tabular}

Table 1. Parameters of S- MAC Implementation on Qualnet 5.0

table 1 that the new modified protocol is better than existing protocols as it provides all the features needed for an efficient MAC design.

\section{Future Scope}

In this paper, different Wireless Sensor Network MAC protocols such as Power Aware Multi-Access with Signaling (PAMAS) protocol, Sensor MAC (S-MAC) protocol, Timeout T-MAC protocol, Wise-MAC protocol, Berkeley Media Access Control (B-MAC) protocol, Low Energy Adaptive Clustering Hierarchy (LEACH) protocol have been discussed. We have drawn the conclusion that the MAC protocol influences network lifetime. However, different MAC protocols can be efficient depending on the given environment and applications. During this work, we realized that the MAC protocols for the wireless sensor networks are a hard and extensive area. Although modification in S-MAC protocol has been proposed, there is possible future work for system performance optimization. Therefore, some of the planned work has to be streamlined away for future work. We see clear paths for future work:

- Verification through employment and wide-ranging simulations.

- Formal descriptions to address other type of MAC protocols and addition of components.

- Cross layer optimization is an area that needs to be explored more extensively.

- Extension of components and formal descriptions to address the other type of WSN MAC Protocols.

\section{References}

[1] Ye, W.; Heidemann, J. ; Estrin, D., “Medium Access Control With Coordinated Adaptive Sleeping for Wireless Sensor Networks", IEEE/ACM Transactions on Networking, Volume: 12, Issue: 3, June 2004, pp:493 - 506.

[2] Akyildiz, Ian F; Weilian Su; Yogesh W.; Sankarasubramaniam ; Cayirci, E ; “A survey on sensor networks", IEEE Communications Magazine:2002, pp.102-114.

[3] Labrador, M. A.; Wightman, P. M., “Topology Control in Wireless Sensor Networks" Springer, USA.

[4] Rugin, R., Mazzini, G., "A simple and efficient MACrouting integrated algorithm for sensor network", IEEE International Conference on Communications, Volume: 6, 20- 24 June 2004, pp: 3499 - 3503.

[5] Zorzi, M., "A new contention-based MAC protocol for geographic forwarding in ad hoc and sensor networks", IEEE International Conference on communications, Vol.6, June 2004, pp: 3481 - 3485.

[6] Brenner, Pablo, "A Technical Tutorial on the IEEE 802.11 Protocol", BreezeCom Wireless Communications, July 1996.

[7] Ghosh, S.; Veeraraghavan, P.; Singh, S.; Zhang, L.; "Performance of a Wireless Sensor Network MAC Protocol with a Global Sleep Schedule" International Journal of Multimedia and Ubiquitous Engineering, Vol. 4, No. 2, April, 2009, pp 99-114.

[8] Ye, W.; Heidemann, J. ; Estrin, D., “An Energy Efficient MAC Protocol for Wireless Sensor Networks", Twenty-First Annual Joint Conference of the IEEE Computer and Communications Societies (INFOCOM) 3 2002, pp- 1567-1576.

[9] IEEE Standard 802.11., “Wireless LAN Medium Access Control (MAC) and Physical Layer (PHY) Specifications", 1999. 